\title{
miR-200b Suppresses Cell Growth, Migration and Invasion by Targeting Notch1 in Nasopharyngeal Carcinoma
}

\author{
Xu Yang ${ }^{a}$ Weichun $\mathrm{Ni}^{\mathrm{a}}$ Ke Lei ${ }^{\mathrm{b}}$ \\ aDepartment of Otorhinolaryngology, Shanghai Xuhui Center Hospital, bepartment of \\ Otorhinolaryngology Head and Neck Surgery, Shanghai 5th Hospital of Fudan University, Shanghai, \\ China
}

\section{Key Words}

miR-200b • Notch1 • Nasopharyngeal carcinoma

\begin{abstract}
Background/Aims: MicroRNAs (miRNAs) are a class of small noncoding RNA molecules that play important roles in carcinogenesis and tumor progression. We investigated the roles and mechanisms of miR-200b in human nasopharyngeal carcinoma (NPC). Methods: We used quantitative real-time polymerase chain reaction (qRT-PCR) analyses to measure levels of miR$200 \mathrm{~b}$ and Notch 1 in NPC specimens and cell lines. Human NPC cell lines stably expressing miR$200 \mathrm{~b}$ or control were used to analyze the tumour-suppressive effect of miR-200b. Luciferase reporter assays were used to determine the association between miR-200b and the Notch1 3' untranslated region. Results: We found that miR-200b is significantly downregulated in NPC tissues and cell lines. Gain-of-function and loss-of-function studies demonstrated that miR$200 \mathrm{~b}$ suppresses NPC cell growth, migration and invasion in vitro. Importantly, overexpression of miR-200b effectively repressed tumor growth in nude mouse models. Integrated analysis identified Notch1 as a direct and functional target of miR-200b. Overexpression of Notch1 reversed the inhibitory effect of miR-200b on NPC cell growth and invasion. Conclusion: These results indicate that miR-200b exerts tumor-suppressive effects in NPC carcinogenesis through the suppression of Notch1 expression and suggest a therapeutic application of miR$200 \mathrm{~b}$ in NPC.
\end{abstract}

\section{Introduction}

Nasopharyngeal carcinoma (NPC) is a form of malignant squamous cell carcinoma which arises from epithelial cells of the nasopharynx. It is unusually prevalent in southern China and in other populations of Cantonese origin, where it has an incidence rate of 20

Ke Lei and Xu Yang
Department of Otorhinolaryngology Head and Neck Surgery, Shanghai 5th Hospital of Fudan University, Shanghai 200240 (China) and Department of Otorhinolaryngology, Shanghai Xuhui Center Hospital, Shanghai 200031 (China)

Tel.+8602164308151, E-Mail keleichina@yeah.net and E-Mail lileyyang@126.com 
to 50 cases per 100,000 population, the highest in the world and approximately 25-fold higher than most other countries [1,2]. NPC is highly malignant and is characterized by local invasiveness and early distant metastases, and once metastasis has occurred the prognosis is very poor [3]. The etiology of NPC has long been known to involve a variety of factors including environmental factors, genetic susceptibility and Epstein-Barr virus (EBV) infection [4], but the underlying molecular mechanisms remain incompletely understood. It is known that a range of factors classified as oncogenes and tumor suppressors are involved in NPC tumorigenesis, and the recently-discovered class of genetic regulators known as micro-RNAs (miRNAs) are now also included in these categories [5, 6].

miRNAs are short, variable, non-coding RNA sequences 19 to 25 nucleotides in length, that act as critical post-transcriptional regulators of gene expression [7]. They act by imperfect base-pair binding to complementary sequences which are generally located within the 3' untranslated region (UTR) of target transcript RNAs and inhibit their translation or reduce their stability andinduce degradation [7]. They have been found to exist in many organisms and to regulate genes involved in a wide range of biological functions including development, differentiation, proliferation and apoptosis [8-11]. There is accumulating evidence that miRNAs are also involved in tumor pathogenesis $[5,6]$, with approximately half of all miRNAs known to date being located within fragile sites and genomic regions associated with cancer. Deregulation of miRNA expression has been associated with carcinogenesis [6, 8], and abnormal expression of miRNAs has been demonstrated in most tumor types [5, 12]. Deregulation of several miRNAs, including miR-26a, miR-29c, miR-100, miR-138, miR-141, miR-200a, miR-216b, and miR-375, has been shown to affect various processes in NPC such as cell growth, apoptosis and metastasis [13-19], and these findings suggest that deregulated miRNA expression is involved in tumorigenesis.

Among the miRNAs reported to be involved in NPC, analysis of NPC tissues in comparison with adjacent normal tissues by Chen et al. using miRNA microarray analysis revealed that miR-200b is downregulated in NPC [20]. However, the role of miR-200b in NPC tumorigenesis remains undefined. In this study, we have characterized miR-200b in NPC and found overexpression of miR-200b suppresses malignant progression of NPC cells by targeting Notch1.

\section{Materials and Methods}

\section{Cell culture and tissue collection}

Five human nasopharyngeal carcinoma cell lines, CNE-1, CNE-2, C666-1, 5-8F and HONE-1, were cultured in RPMI-1640 medium (Invitrogen, Carlsbad, CA) supplemented with $10 \%$ fetal bovine serum (FBS). The immortalized nonmalignant human nasopharyngeal epithelial cell line NP69 was cultured in keratinocyte-serum free medium (Invitrogen) supplemented with bovine pituitary extract (BD Biosciences, San Jose, CA) [17, 21]. NPC samples and non-tumor nasopharyngeal epithelial tissues were collected from Shanghai 5th Hospital of Fudan University (Shanghai, China). None of the NPC patients had undergone radiotherapy or chemotherapy before biopsy. All patients provided written informed consent. The studies using human tissues were reviewed and approved by the Committee for Ethical Review of Research Involving Human Subjects at Fudan University.

\section{RNA isolation and quantitative real-time PCR (qRT-PCR)}

Total RNA was extracted using TRIzol reagent (Invitrogen). To measure expression of miR-200b, RNA was reverse transcribed using Bulge-Loop ${ }^{\mathrm{TM}}$ microRNA specific RT primers (RiboBio, Guangzhou, China) and M-MLV reverse transcriptase (Promega, Madison, WI). To measure mRNA expression of Notch1, total RNA was reverse transcribed using random primers and M-MLV reverse transcriptase. Quantitative PCR was performed on a PRISM 7900HT system (Applied Biosystems. Foster City, CA) using Platinum SYBR Green qPCR SuperMix-UDG reagents (Invitrogen) [17]. The snRNA U6 or $\beta$-actin were used as normalization controls and the relative expression levels were calculated using the $2-^{-\Delta \Delta c t}$ method [22]. 


\section{Lentivirus transductionand oligonucleotide transfection}

miR-200b was purchased from Origin (Beijing, China). Constructs containing the pre-miR-200b sequence together with 100 bases of the upstream and downstream flanking sequences were cloned into the pGCSIL-GFP vector. C666-1 or 5-8F cells $\left(5 \times 10^{5}\right)$ were infected with $5 \times 10^{7}$ lentiviral units in the presence of $10 \mu \mathrm{g} / \mathrm{mL}$ polybrene. Empty lentiviral vector was used as the negative control. Both miR200b inhibitor and negative control were synthesized by RiBo Bio (Guangzhou, China). Transfection with inhibitor or negative control was performed using Lipofectamine 2000 (Invitrogen) for $48 \mathrm{~h}$, according to the manufacturer's instructions.

\section{Plasmid construction and luciferase reporter assays}

A 487-bp fragment from the Notch1 3'UTR (position 509-995) containing the binding sequences of miR-200b was amplified by PCR from a human cDNA library. The fragment was then cloned into the pGL3control vector (Promega) downstream of the firefly luciferase gene. The predicted miR-200b binding site was mutated using the QuikChange II XL Site-Directed Mutagenesis Kit (Stratagene, La Jolla, CA). C6661 or $5-8 \mathrm{~F}$ cells were cotransfected with pGL3-Notch1 or pGL3-control vector, together with miR-200b or control vector. After $48 \mathrm{~h}$ of culture, the transfected cells were assayed using the dual luciferase reporter assay system (Promega) with a MicroLumatPlus LB96V luminometer (Berthold Technologies, Bad Wildbad, Germany).

\section{Cell proliferation assays}

Cell proliferation was assessed using the MTT cell viability assay. Following transfection for $48 \mathrm{~h}$, cells were collected and seeded into new 96-well plates at 2,000 cells/well then cultured for 24, 48, 72 or $96 \mathrm{~h}$. MTT solution was then added to each well to a final concentration of $0.2 \mathrm{mg}$ MTT $/ \mathrm{mL}$ and the plates were incubated at $37^{\circ} \mathrm{C}$ for a further $1 \mathrm{~h}$. At the end of the incubation the formazan was dissolved by the addition of $200 \mu \mathrm{L}$ dimethyl sulfoxide and the absorbance was measured at $450 \mathrm{~nm}$ using a spectrophotometer.

\section{Cell migration and invasion assays}

Wound healing assays were used to examine migration of NPC cells. C666-1 or 5-8F cells, untransfected or transfected with miR-200b, were cultured until confluent and then a sterile $200 \mu \mathrm{L}$ pipette tip was used to scrape a clear line through the cell layer. Cultures were returned to the incubator for a further $48 \mathrm{~h}$, at the end of which the cultures were photographed under a light microscope and the distance between the migration fronts was measured. Migration rate was expressed as the percentage wound closure [23]. To quantify invasion, $1 \times 10^{5}$ C666-1 or 5-8F cells, untreated or transfected with miR-200b or anti-miR-200b as shown, were plated into a BD Biocoat ${ }^{\mathrm{TM}}$ Matrigel $^{\mathrm{TM}}$ invasion chamber (BD Biosciences) consisting of a multiwell plate with cell culture inserts containing an 8 micron pore size PET membrane overlaid with a thin layer of Matrige ${ }^{\mathrm{TM}}$ basement membrane matrix which blocks non-invasive cells from migrating through the membrane. FBS was added to the lower wells as a chemoattractant and the plates were incubated for 48 $\mathrm{h}$, at the end of which non-invading cells were removed from the upper surface with a cotton swab. Invasive cells on the lower side of the membrane were then stained with crystal violet, air dried and photographed, and the number of stained cells was counted [23].

\section{Western blotting}

Cells were lysed in lysis buffer (Cell Signaling Technology, Danvers, MA) containing $1 \mathrm{mM}$ phenylmethylsulfonyl fluoride (Sigma-Aldrich, St Louis, MO) and incubated on ice for $10 \mathrm{~min}$. Lysates were then centrifuged at $12,000 \mathrm{rpm}$ at $4^{\circ} \mathrm{C}$ for $10 \mathrm{~min}$ and the protein concentration of the supernatant was quantitated using the Bradford assay (BioRad, Hercules, CA). Aliquots containing $30 \mu \mathrm{g}$ of protein were resolved by SDS-PAGE and electroblotted onto PVDF membranes (Millipore, Bedford, MA). The expression of Notch1 and $\beta$-actin were detected using specific anti-Notch1 (Santa Cruz Biotechnology, Santa Cruz, CA) and anti- $\beta$-actin (GE Healthcare, Waukesha, WI) antibodies.

\section{Tumor growth assays}

The experimental protocol and animal handling procedures were approved by the institutional animal ethics committee. To assess tumor growth, C666-1 cells stably expressing miR-200b or control were injected subcutaneously into the dorsal flank of female BALB/c nude mice aged 4 to 5 weeks ( $n=5$ per group). The 
A

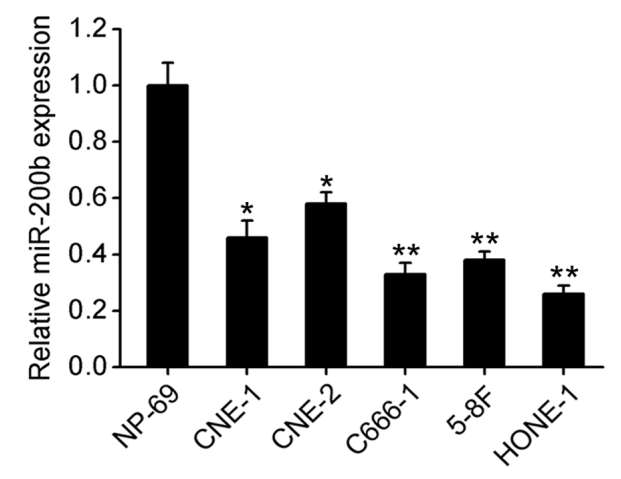

$\mathrm{B}$

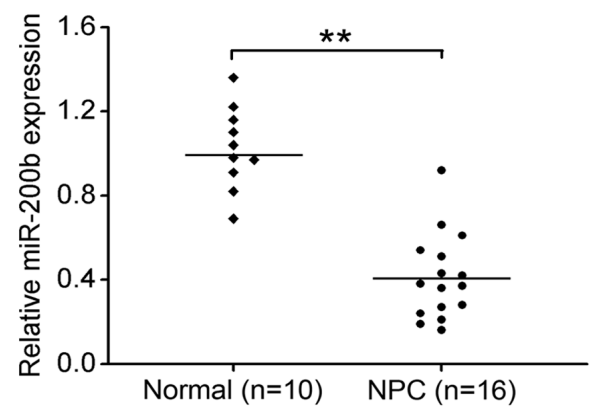

Fig. 1. The expression of miR-200b in NPC cell lines and clinical specimens. (A) Expression levels of miR200b were quantified by qRT-PCR in NP69 cells and five NPC cell lines. U6 snRNA was used as an internal control. Data are presented as the mean \pm SD from 3 replicate samples. ${ }^{*} P<0.05,{ }^{*} P<0.01$ vs. NP-69. (B) Expression levels of miR-200b in NPC tissues $(n=16)$ and normal nasopharyngeal epithelial tissues $(\mathrm{n}=10) .{ }^{* *} P<0.01$.

mice were observed over 4 weeks for tumor formation. After 25 days, the mice were euthanized and the tumors were removed, photographed, weighed and measured. Tumor volumes were calculated as follows: volume $=\left(D \times d^{2}\right) / 2$, where $D$ represented the longest diameter and $d$ the shortest.

\section{Statistical analysis}

SPSS 13.0 software was used for statistical analysis. Data are expressed as the mean \pm SD from at least three independent experiments. Differences between groups were analyzed using Student's $t$-test when comparing only two groups, while one-way analysis of variance (ANOVA) was used when comparing more than two groups. The association between miR-200b and Notch1 expression was evaluated using Spearman's correlation analysis. Statistical differences with $P<0.05$ were considered significant.

\section{Results}

miR-200b is downregulated in NPC cell lines and clinical specimens

To confirm the previous finding by Chen et al. [20] that miR-200b is downregulated in NPC, we quantified miR-200b expression in five NPC cell lines by qRT-PCR and compared this with expression in the nonmalignant nasopharyngeal epithelial cell line NP69. As shown in Fig. 1A, miR-200b expression was significantly reduced in all five carcinoma cell lines in comparison to NP69 cells. We then analyzed the expression of miR-200b in 16 primary NPC specimens and 10 normal nasopharyngeal epithelial tissues. The results showed that miR$200 \mathrm{~b}$ was significantly downregulated in NPC samples compared to normal tissues (Fig. 1B). These findings provide novel evidence for downregulation of miR-200b in human NPC cell lines and clinical specimens.

\section{miR-200b inhibits NPC cell growth in vitro and in vivo}

Since reduced expression of miR-200b was observed in malignant cells, we then investigated whether restoration of miR-200b was capable of inhibiting the growth of these cells. Both C666-1 and 5-8F NPC cell lines were infected with control lentivirus or lentivirus carrying miR-200b, and increased expression of miR-200b in the miR-200b-infected cells was confirmed by qRT-PCR (Fig. 2A). Using the MTT assay as a measure of cell viability, we found that overexpression of miR-200b significantly inhibited cell viability, which was assumed to reflect cell numbers in the cultures and thus proliferation, while blocking miR- 


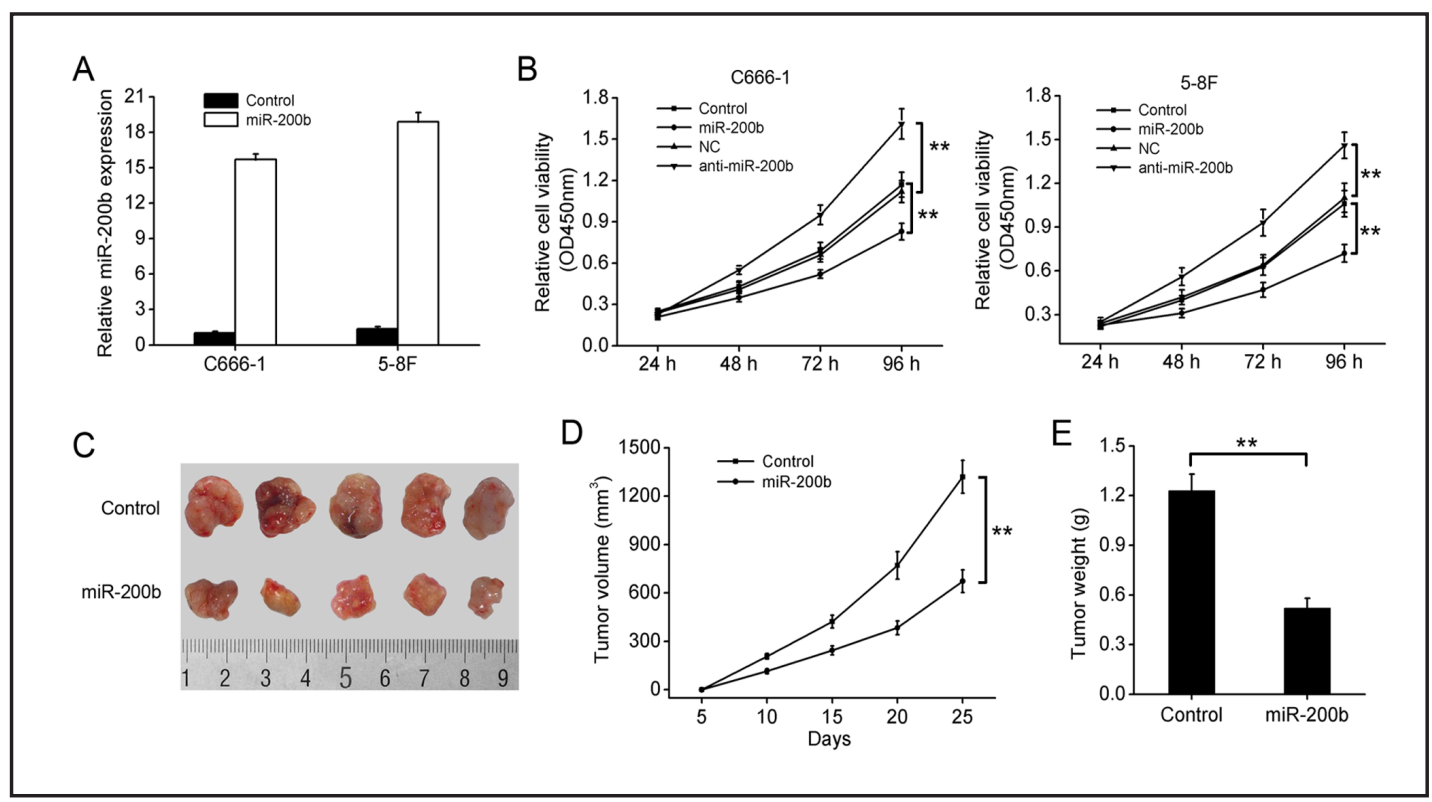

Fig. 2. miR-200b inhibits NPC cell growth in vitro and in vivo. (A) C666-1 and 5-8F cells were infected with miR-200b lentivirus (miR-200b) or control lentivirus (Control), respectively, and the expression of miR200b was determined by qRT-PCR. (B) Effect of miR-200b on cell proliferation was measured by MTT assay in C666-1 and 5-8F cells infected with miR-200b lentivirus or transfected with anti-miR-200b. The negative control of anti-miR-200b was indicated as NC. Data are presented as the mean \pm SD from 3 experiments. (C) miR-200b-overexpressing or control C666-1 cells were injected subcutaneously into BALB/c nude mice. After 25 days, the mice were euthanized and the tumors were recovered and photographed. (D) The growth curve of tumor volumes. (E) The weight of xenograft tumors. Data represent mean \pm SD from 5 nude mice. $* P<0.05,{ }^{* *} P<0.01$.

200b with anti-miR-200b significantly increased cell viability over the same time period (Fig. 2B). To investigate whether the inhibition of cell growth by miR-200b was also effective in vivo, miR-200b-overexpressing or control C666-1 cells were injected subcutaneously into the flanks of BALB/c nude mice. After 25 days, mice were sacrificed and the resulting tumors were removed for analysis. The results showed that both tumor volume and weight were significantly reduced when miR-200b-overexpressing cells were implanted, compared to control C666-1 cells (Fig. 2C-E). These results implied that miR-200b has a strong ability to suppress NPC cell growth.

\section{miR-200b inhibits NPC cell migration and invasion in vitro}

We next examined the effect of miR-200b on NPC cell migration and invasion. Using a wound-healing assay, we found that overexpression of miR-200b caused a suppression of cell migration in the C666-1 and 5-8F cell lines (Fig. 3A). In contrast, the migration of these cells increased when endogenous miR-200b was knockdown (Fig. 3B). To assess invasiveness of the NPC cell lines, we used the Matrigel transwell invasion assays. As shown in Fig. 3C, overexpression of miR-200b markedly reduced the invasive ability of C666-1 and 5-8F cells. Conversely, transfection with anti-miR-200b significantly increased cell invasiveness (Fig. $3 \mathrm{D})$. These results suggest that miR-200b can significantly suppress in vitro migration and invasion of NPC cells.

miR-200b directly targets Notch1 3'UTR

To determine the targets of miR-200b, we screened a large number of potential target proteins in a database library to identify promising miRNA binding seed sequences within 3' untranslated region (UTR). Using bioinformatic analyses (Targetscan, microRNA.org), we 
A
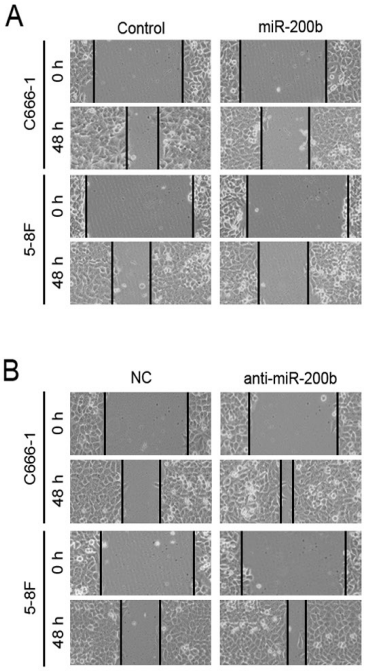

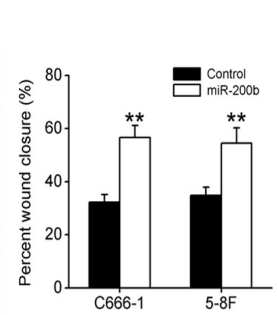

C
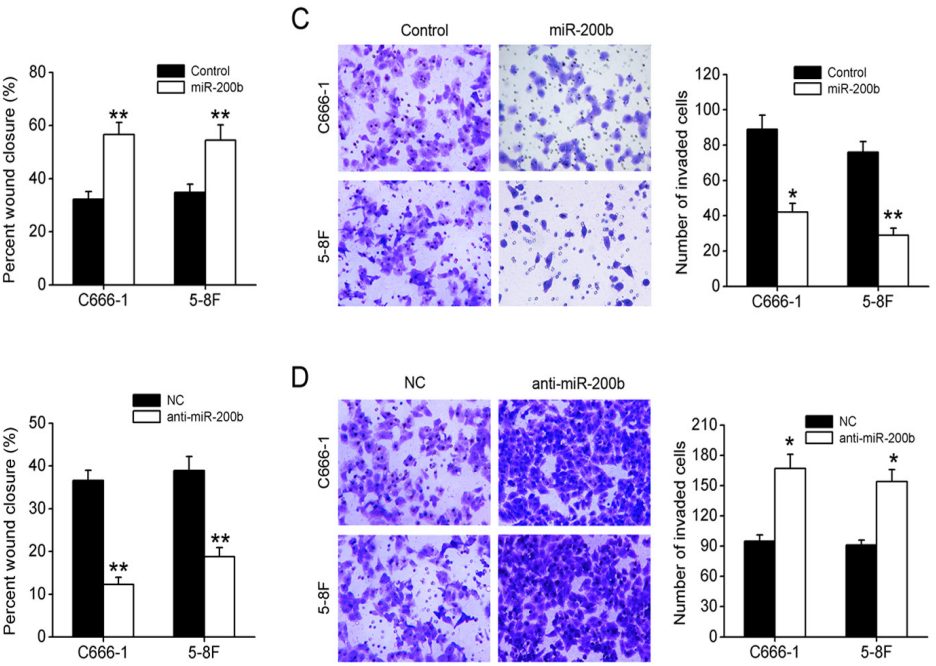

D

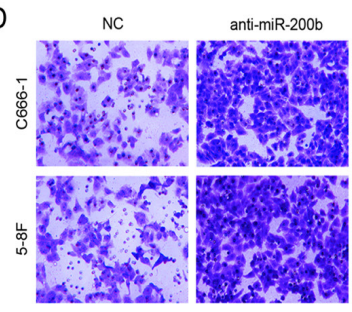

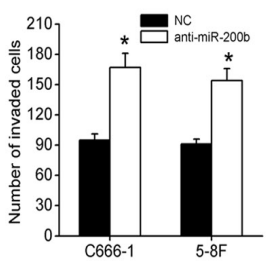

Fig. 3.miR-200b inhibits NPC cell migration and invasion in vitro. (A) The wound healing rate was significantly decreased in miR-200b-overexpressing C666-1 and 5-8F cells as compared with control. (B) The wound healing rate was significantly increased in C666-1 and 5-8F cells transfected with anti-miR-200b compared with the negative control NC. (C) Transwell invasion assays using C666-1 and 5-8F cells stably expressing miR-200b or control. (D) Transwell invasion assays using C666-1 and 5-8F cells transiently transfected with anti-miR-200b or NC. Data are presented as the mean \pm SD from 3 experiments. ${ }^{*} P<0.05$, ${ }^{*} P<0.01$ vs. control.
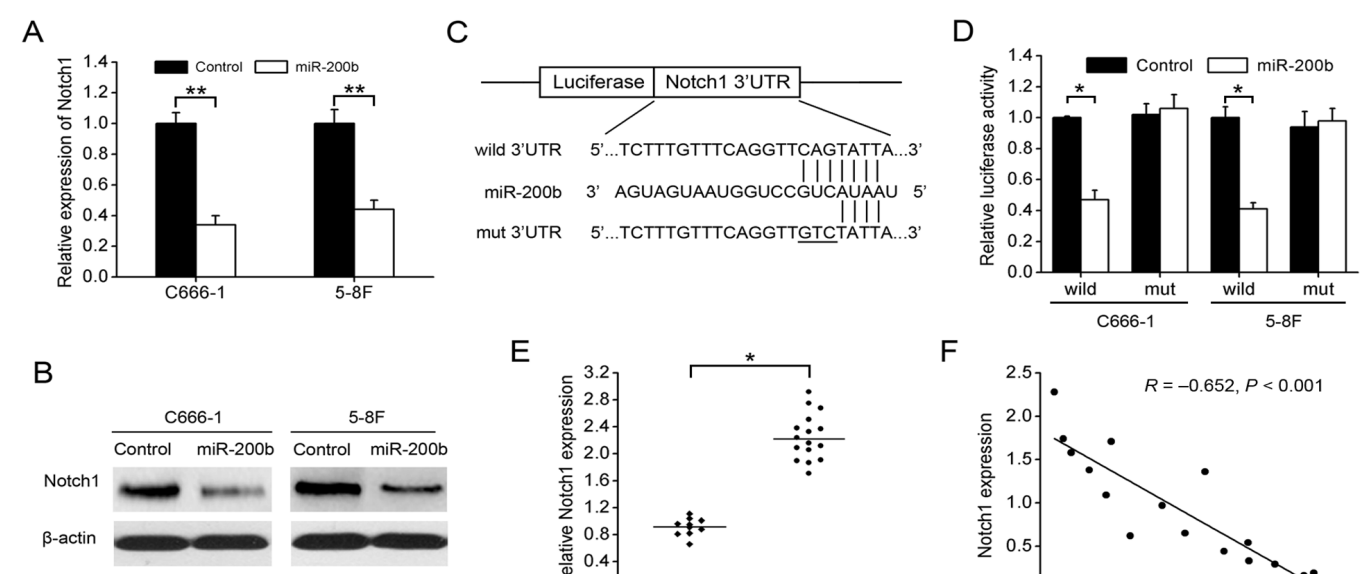

$\mathrm{E}$

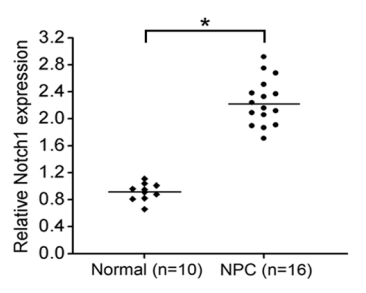

$\mathrm{F}$

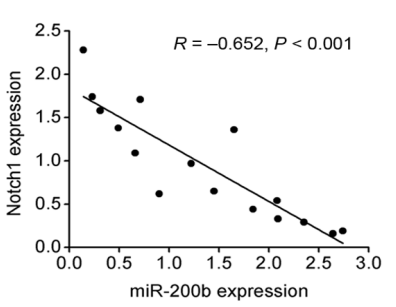

Fig. 4. Notch1 is a downstream target of miR-200b. (A) Expression levels of Notch1 mRNA in C666-1 and 5-8F cells stably expressing miR-200b were analyzed by qRT-PCR. (B) Expression levels of Notch1 protein in C666-1 and 5-8F cells stably expressing miR-200b were analyzed by western blotting. (C) A schematic diagram of Notch1 3'UTR containing reporter constructs. (D) Luciferase reporter assays in C666-1 and 5-8F cells, following cotransfection of cells with the wild-type or mutant 3'UTR Notch1 and miR-200b. (E) Average expression level of Notch1 mRNA in NPC tissues and normal nasopharyngeal tissues. (F) Correlation of miR-200b to Notch1 mRNA levels in 16 NPC tissues using Spearman's correlation analysis. Expression was analyzed by qRT-PCR and normalized to expression of U6 snRNA. Data are presented as the mean \pm SD from 3 experiments. ${ }^{*} P<0.05,{ }^{* *} P<0.01$.

found that Notch1 is a potential target for miR-200b, as Notch signaling is closely associated with tumorigenesis and cell invasion [24]. We first investigated expression of Notch1 in NPC cells with or without overexpression of miR-200b. In C666-1 and 5-8F cells, using qRT-PCR 


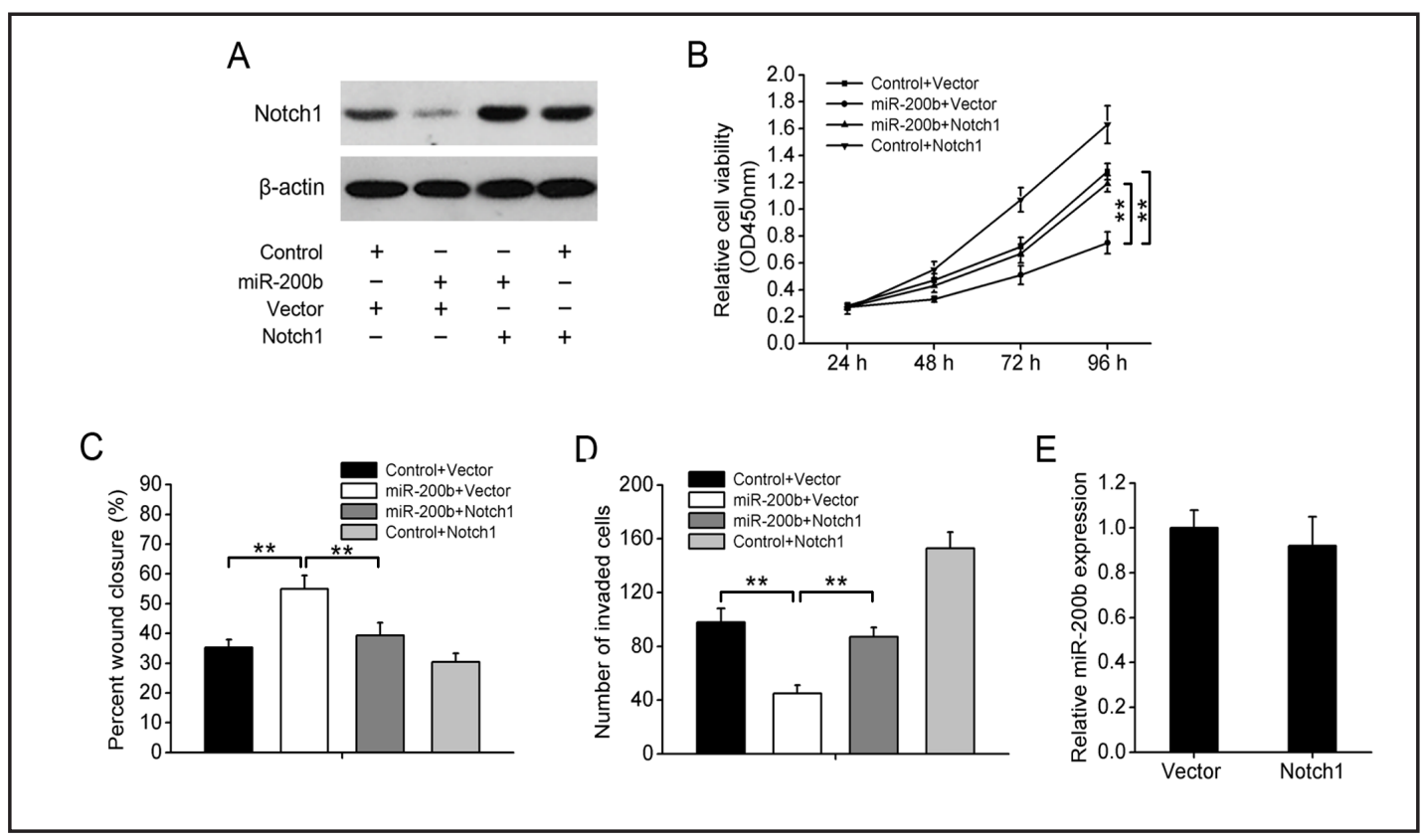

Fig. 5. Notch 1 can reverse the inhibitory effect of miR-200b on NPC cell growth and invasion. (A) Western blotting analysis of Notch1 levels in C666-1 cells transfected with the Notch1 plasmid (without 3'UTR) or vector control or Notch1 transfection, in combination with miR-200b transduction. (B) MTT cell viability assays. (C) Wound healing assays. (D) Transwell invasion assays. (E) qRT-PCR analysis of miR-200b expression. Data are presented as the mean \pm SD from 3 experiments. ${ }^{*} P<0.05,{ }^{* *} P<0.01$.

and western blotting, we found that both mRNA and protein expression of Notch1 were reduced by overexpression of miR-200b (Fig. 4A and B). We then used a luciferase assay to investigate whether miR-200b can functionally interact directly with the putative binding site in the 3'UTR of Notch1, using the reporter construct shown in Fig. 4C. In both C666-1 and $5-8$ F cells, cotransfection of the cells with wild or mutant Notch1 3'UTR together with miR$200 \mathrm{~b}$ or control lentivirus showed that miR-200b interacts with the wild Notch1 3'UTR to reduce luciferase expression (Fig. 4D). Subsequently, we analyzed the expression of Notch1 in NPC tissues and normal nasopharyngeal epithelium. Notch1 expression was found to be significantly higher in NPC tissues compared to normal samples (Fig. 4E), and analysis of miR-200b and Notch1 expression in 16 NPC specimens by Spearman's correlation analysis showed a significant inverse correlation $(R=-0.652, P<0.001$; Fig. $4 \mathrm{~F})$.

Notch1can reverse the inhibitory effect of miR-200b on NPC cell growth, migration and invasion

Since our results support the hypothesis that miR-200b acts via downregulation of Notch1 to inhibit cell growth and invasion, we tested whether Notch1 is a direct functional mediator of the inhibitory effect of miR-200b. C666-1 cells were transfected with Notch1 plasmid without the 3'UTR or control plasmid, in combination with miR-200b or control lentivirus transduction. The Notch1 construct without the 3'UTR is insensitive to miR-200bmediated repression. Western blotting of the resultant cultures confirmed that miR-200b downregulated Notch1 expression, but that this could be restored by transfection with Notch1 plasmid (Fig. 5A). Using the MTT assay, we observed that overexpression of Notch1 significantly rescued miR-200b-induced cell growth inhibition (Fig. 5B). Furthermore, miR-200b overexpression significantly reduced C666-1 cell migration and invasion, while transfection with Notch1 reversed this inhibitory effect (Fig. 5C and D). These results suggest that Notch1 is responsible for mediating the effects of miR-200b on NPC cell growth, migration and invasion. 


\section{Discussion}

In this study, we confirm that miR-200b is downregulated in NPC tissues and cell lines. We demonstrate that miR-200b regulates proliferation and invasion of NPC cells, suggesting that it may play a key role in tumorigenesis and metastasis.

Our initial analysis of miR-200b expression by qRT-PCR revealed that miR-200b expression was significantly downregulated in all the NPC cell lines tested, compared to the nonmalignant immortalized nasopharyngeal cell line NP69. To confirm that these results held true in nontransformed cells, we tested primary tissues from 16 cases of NPC and compared them with 10 normal tissue samples, and found that miR-200b expression was again significantly reduced in NPC. The data were similar to the findings in gastric cancer and glioma, in which miR-200b was downregulated $[25,26]$. However, several other reports showed that miR-200b was upregulated in ovarian and bladder cancer $[27,28]$. These controversial results demonstrated that the role of miR-200b was possibly tumor specific and highly dependent on its targets in different cancer cells.

We next investigated whether this downregulation of miR-200b was responsible for the uncontrolled growth of tumor cells. First we confirmed that transduction of NPC cells with miR-200b lentivirus resulted in increased miR-200b expression. We then analyzed cell growth in vitro and in vivo, and found that both cell growth and tumor growth were suppressed in cells overexpressing miR-200b. We also found that expression of miR-200b reduced the invasive potential of NPC cells, while anti-miR-200b increased invasiveness. These findings support a role of miR-200b downregulation in allowing uncontrolled growth of tumor cells as well as anchorage-independent growth, a prerequisite for metastasis.

Previous studies have shown that expression of miRNAs is altered in many types of cancer, and have identified a variety of miRNA targets. These include EZH2 which is a target of miR-26a in rhabdomyosarcoma and lymphoma $[16,29,30]$, miR-29c which is downregulated in NPC cells and suppresses NPC cell migration and invasion by targeting TIAM1 [17], miR-125b which suppresses liver cancer cell proliferation and metastasis by targeting oncogene LIN28B [31], and miR-124 which has been shown to have an antiproliferative role in various cancers including cervical cancer and meduloblastoma [14, 32, 33 and to inhibit cell proliferation in gastric cancer through down-regulation of SPHK-1 [14]. Consequently we aimed to elucidate the role of miR-200b in NPC and to investigate and identify a potential intermediary.

Notch family members are important in controlling cell growth and invasion, and as such they play an important role in early embryonic development $[34,35]$. Notch signaling involves a complex array of activator and repressor genes which are not yet completely understood, and activates a wide range of targets [36]. Notch1 has also been shown to be a target of several miRNAs, including miR-34a and miR-146a, through which these miRNAs regulate cancer cell proliferation, differentiation and invasion [24, 37, 38]. In this study, we investigated whether miR-200b acts via targeting of Notch1 in NPC. Using a luciferase reporter assay, we demonstrate conclusively that miR-200b directly targets Notch 1 by binding to the potential 3'UTR binding site. Through this miR-200b acts to suppress growth and invasion of NPC cells.

It is worth noting that a single miRNA can influence the expression of various transcripts that have important roles in cancer development and progression [39]. Previous studies have reported several functional targets of miR-200b in cancer-related processes, including CREB1 in cell growth and ZEB2 in cell migration and invasion $[25,26]$. Recently, Tang et $a l$. have reported that ectopic expression of miR-200b and miR-200c impaired cell growth and invasion by directly targeting DNMT3A, DNMT3B and SP1 [40]. Zhang et al. found that miR-200b suppressed invasiveness and modulated the cytoskeletal and adhesive machinery in esophageal squamous cell carcinoma cells via targeting Kindlin-2 [41]. Our study adds Notch1 to the growing group of miR-200b target genes and provides evidence that miR200b suppresses NPC cell growth, migration and invasion by repressing Notch1 expression. 
On the other hands, according to Bao and colleagues' findings that over-expression of Notch1 leads to decreased expression of miR-200b, miR-200c, let-7a, let-7b, and let-7c in pancreatic cancer cells [42], we examined miR-200b expression levels in NPC cells stably expressing Notch1. The results showed that overexpression of Notch1 had no effect on miR200b expression levels (Fig. 5E). However, we found that Notch1 overexpression increased NPC cell growth, migration and invasion compared with Notch1 and miR-200b group (Fig. 5B-D). Perhaps Notch1 affected other tumor-associated genes and this need further studies to clarify. In conclusion, our results indicate that miR-200b is important in regulating tumor growth and may play a key role in controlling the initiation of metastasis. Targeting Notch1, it thus indirectly regulates a wide range of other genes involved in cell growth and oncogenesis, while Notch1 regulation of miR-200b constitutes a putative positive feedback loop. This regulatory system may provide novel therapeutic targets in NPC and other cancers, allowing intervention before the disease progresses to a stage at which treatment options are extremely limited.

\section{Conflict of Interest}

All authors are in agreement with the content of this manuscript. The authors declare no conflict of interest.

\section{Acknowledgements}

This study was supported by Shanghai Clinical Research Center, Chinese Academy of Science (Grant No. CCR2012004).

\section{References}

$>_{2}$

$>2$

$\checkmark 3$

$>4$

$-5$

- 6

$-7$

$-8$

$>9$

-10
-11

$-12$

$>13$

Yu MC, Yuan JM: Epidemiology of nasopharyngeal carcinoma. Semin Cancer Biol 2002;12:421-429. Jemal A, Bray F, Center MM, Ferlay J, Ward E, Forman D: Global cancer statistics. CA Cancer J Clin 2011;61:69-90.

Lai SZ, Li WF, Chen L, Luo W, Chen YY, Liu LZ, Sun Y, Lin AH, Liu MZ, Ma J: How does intensity-modulated radiotherapy versus conventional two-dimensional radiotherapy influence the treatment results in nasopharyngeal carcinoma patients? Int J Radiat Oncol Biol Phys 2011;80:661-668.

Clifford P: A review on the epidemiology of nasopharyngeal carcinoma. Int J Cancer 1970;5:287-309.

Calin GA, Croce CM: MicroRNAs and chromosomal abnormalities in cancer cells. Oncogene 2006;25:62026210.

Esquela-Kerscher A, Slack FJ: Oncomirs - microRNAs with a role in cancer. Nat Rev Cancer 2006;6:259-269. Bartel DP: MicroRNAs: genomics, biogenesis, mechanism, and function. Cell 2004;116:281-297.

Ambros V: MicroRNA pathways in flies and worms: growth, death, fat, stress, and timing. Cell 2003;113:673-676.

Ambros V: The functions of animal microRNAs. Nature 2004;431:350-355.

Croce CM, Calin GA: miRNAs, cancer, and stem cell division. Cell 2005;122:6-7. Bushati N, Cohen SM: microRNA functions. Annu Rev Cell Dev Biol 2007;23:175-205.

Lu J, Getz G, Miska EA, Alvarez-Saavedra E, Lamb J, Peck D, Sweet-Cordero A, Ebert BL, Mak RH, Ferrando AA, Downing JR, Jacks T, Horvitz HR, Golub TR: MicroRNA expression profiles classify human cancers. Nature 2005;435:834-838.

Shi W, Alajez NM, Bastianutto C, Hui AB, Mocanu JD, Ito E, Busson P, Lo KW, Ng R, Waldron J, O'Sullivan B, Liu FF: Significance of Plk1 regulation by miR-100 in human nasopharyngeal cancer. Int J Cancer 2010;126:2036-2048. 
-14 Xia J, Wu Z, Yu C, He W, Zheng H, He Y, Jian W, Chen L, Zhang L, Li W: miR-124 inhibits cell proliferation in gastric cancer through down-regulation of SPHK1. J Pathol 2012;227:470-480.

-15 Hui AB, Bruce JP, Alajez NM, Shi W, Yue S, Perez-Ordonez B, Xu W, O'Sullivan B, Waldron J, Cummings B, Gullane P, Siu L, Liu FF: Significance of dysregulated metadherin and microRNA-375 in head and neck cancer. Clin Cancer Res 2011;17:7539-7550.

16 Lu J, He ML, Wang L, Chen Y, Liu X, Dong Q, Chen YC, Peng Y, Yao KT, Kung HF, Li XP: MiR-26a inhibits cell growth and tumorigenesis of nasopharyngeal carcinoma through repression of EZH2. Cancer Res 2011;71:225-233.

-17 Liu N, Tang L-L, Sun Y, Cui R-X, Wang H-Y, Huang B-J, He QM, Jiang W, Ma J: MiR-29c suppresses invasion and metastasis by targeting TIAM1 in nasopharyngeal carcinoma. Cancer Lett 2013;329:181-188.

18 Liu X, Lv XB, Wang XP, Sang Y, Xu S, Hu K, Wu M, Liang Y, Liu P, Tang J, Lu WH, Feng QS, Chen LZ, Qian CN, Bei JX, Kang T, Zeng YX: MiR-138 suppressed nasopharyngeal carcinoma growth and tumorigenesis by targeting the CCND1 oncogene. Cell Cycle 2012;11:2495-2506.

19 Deng M, Tang H, Zhou Y, Zhou M, Xiong W, Zheng Y, Ye Q, Zeng X, Liao Q, Guo X, Li X, Ma J, Li G: miR216b suppresses tumor growth and invasion by targeting KRAS in nasopharyngeal carcinoma. J Cell Sci 2011;124:2997-3005.

20 Chen HC, Chen GH, Chen YH, Liao WL, Liu CY, Chang KP, Chang YS, Chen SJ: MicroRNA deregulation and pathway alterations in nasopharyngeal carcinoma. Br J Cancer 2009;100:1002-1011.

21 Tsao SW, Wang X, Liu Y, Cheung YC, Feng H, Zheng Z, Wong N, Yuen PW, Lo AK, Wong YC, Huang DP: Establishment of two immortalized nasopharyngeal epithelial cell lines using SV40 large T and HPV16E6/ E7 viral oncogenes. Biochim Biophys Acta 2002;1590:150-158.

-22 Livak KJ, Schmittgen TD: Analysis of relative gene expression data using real-time quantitative PCR and the 2(-Delta Delta C(T)) Method. Methods 2001;25:402-408.

-23 Zheng F, Liao YJ, Cai MY, Liu YH, Liu TH, Chen SP, Bian XW, Guan XY, Lin MC, Zeng YX, Kung HF, Xie D: The putative tumour suppressor microRNA-124 modulates hepatocellular carcinoma cell aggressiveness by repressing ROCK2 and EZH2. Gut 2012;61:278-289.

24 Pang RT, Leung CO, Ye TM, Liu W, Chiu PC, Lam KK, Lee KF, Yeung WS: MicroRNA-34a suppresses invasion through downregulation of Notch1 and Jagged1 in cervical carcinoma and choriocarcinoma cells. Carcinogenesis 2010;31:1037-1044.

-25 Kurashige J, Kamohara H, Watanabe M, Hiyoshi Y, Iwatsuki M, Tanaka Y, Kinoshita K, Saito S, Baba Y, Baba $\mathrm{H}$ : MicroRNA-200b regulates cell proliferation, invasion, and migration by directly targeting ZEB2 in gastric carcinoma. Ann Surg Oncol 2012;19:S656-664.

26 Peng B, Hu S, Jun Q, Luo D, Zhang X, Zhao H, Li D: MicroRNA-200b targets CREB1 and suppresses cell growth in human malignant glioma. Mol Cell Biochem 2013;379:51-58.

27 Iorio MV, Visone R, Di Leva G, Donati V, Petrocca F, Casalini P, Taccioli C, Volinia S, Liu CG, Alder H, Calin GA, Ménard S, Croce CM: MicroRNA signatures in human ovarian cancer. Cancer Res 2007;67:8699-8707.

28 Pignot G, Cizeron-Clairac G, Vacher S, Susini A, Tozlu S, Vieillefond A, Zerbib M, Lidereau R, Debre B, Amsellem-Ouazana D, Bieche I: microRNA expression profile in a large series of bladder tumors: identification of a 3-miRNA signature associated with aggressiveness of muscle-invasive bladder cancer. Int J Cancer 2013;132:2479-2491.

-29 Ciarapica R, Russo G, Verginelli F, Raimondi L, Donfrancesco A, Rota R, Giordano A: Deregulated expression of miR-26a and Ezh2 in rhabdomyosarcoma. Cell Cycle 2009;8:172-175.

-30 Sander S, Bullinger L, Klapproth K, Fiedler K, Kestler HA, Barth TF, Möller P, Stilgenbauer S, Pollack JR, Wirth T: MYC stimulates EZH2 expression by repression of its negative regulator miR-26a. Blood 2008;112:4202-4212.

-31 Liang L, Wong CM, Ying Q, Fan DN, Huang S, Ding J, Yao J, Yan M, Li J, Yao M, Ng IO, He X: MicroRNA125b suppressesed human liver cancer cell proliferation and metastasis by directly targeting oncogene LIN28B2. Hepatology 2010;52:1731-1740.

-32 Wilting SM, van Boerdonk RA, Henken FE, Meijer CJ, Diosdado B, Meijer GA, le Sage C, Agami R, Snijders PJ, Steenbergen RD: Methylation-mediated silencing and tumour suppressive function of hsa-miR-124 in cervical cancer. Mol Cancer 2010;9:167.

-33 Pierson J, Hostager B, Fan R, Vibhakar R: Regulation of cyclin dependent kinase 6 by microRNA 124 in medulloblastoma. J Neurooncol 2008;90:1-7. 
-34 34 Cobellis L, Mastrogiacomo A, Federico E, Schettino MT, De Falco M, Manente L, Coppola G, Torella M, Colacurci N, De Luca A: Distribution of Notch protein members in normal and preeclampsia-complicated placentas. Cell Tissue Res 2007;330:527-534.

-35 Sahin Z, Acar N, Ozbey 0, Ustunel I, Demir R: Distribution of Notch family proteins in intrauterine growth restriction and hypertension complicated human term placentas. Acta Histochem 2011;113:270-276.

-36 Wang Z, Ahmad A, Li Y, Azmi AS, Miele L, Sarkar FH: Targeting notch to eradicate pancreatic cancer stem cells for cancer therapy. Anticancer Res 2011;31:1105-1113.

-37 Li WB, Ma MW, Dong LJ, Wang F, Chen LX, Li XR: MicroRNA-34a targets notch1 and inhibits cell proliferation in glioblastoma multiforme. Cancer Biol Ther 2011;12:477-483.

38 Mei J, Bachoo R, Zhang CL: MicroRNA-146a inhibits glioma development by targeting Notch1. Mol Cell Biol 2011;31:3584-3592.

39 Filipowicz W, Bhattacharyya SN, Sonenberg N: Mechanisms of post-transcriptional regulation by microRNAs: are the answers in sight? Nat Rev Genet 2008;9:102-114.

40 Tang H, Deng M, Tang Y, Xie X, Guo J, Kong Y, Ye F, Su Q Xie X: miR-200b and miR-200c as prognostic factors and mediators of gastric cancer cell progression. Clin Cancer Res 2013;19:5602-5612.

41 Zhang HF, Zhang K, Liao LD, Li LY, Du ZP, Wu BL, Wu JY, Xu XE, Zeng FM, Chen B, Cao HH, Zhu MX, Dai LH, Long L, Wu ZY, Lai R, Xu LY, Li EM: miR-200b suppresses invasiveness and modulates the cytoskeletal and adhesive machinery in esophageal squamous cell carcinoma cells via targeting Kindlin-2. Carcinogenesis DOI 10.1093/carcin/bgt320.

42 Bao B, Wang Z, Ali S, Kong D, Li Y, Ahmad A, Banerjee S, Azmi AS, Miele L, Sarkar FH: Notch-1 induces epithelial-mesenchymal transition consistent with cancer stem cell phenotype in pancreatic cancer cells. Cancer Lett 2011;307:26-36. 